4. Landman D, Babu E, Shah N, et al. Transmission of carbapenemresistant pathogens in New York City hospitals: progress and frustration. J Antimicrob Chemother 2012;67:1427-1431.

5. Hospital-acquired infection, New York State, 2014. New York State Department of Health website. https://www.health.ny. gov/statistics/facilities/hospital/hospital_acquired_infections/2014/ docs/hospital_acquired_infection.pdf. Published 2014. Accessed December 13, 2017.

6. Guh AY, Bulens SN, Mu Y, et al. Epidemiology of carbapenemresistant Enterobacteriaceae in 7 US communities, 2012-2013. JAMA 2015;314:1479-1487.

7. Van Duin D, Doi Y. The global epidemiology of carbapenemaseproducing. Enterobacteriaceae. Virulence 2017;8:460-469.

8. Jin Y, Shao C, Li J, Fan H, Bai Y, Wang Y. Outbreak of multidrug resistant NDM-1-producing Klebsiella pneumoniae from a neonatal unit in Shangdong Province, China. PLOS One 2015. doi: 10.1371/journal.pone.0119571.

9. Akturk H, Sutcu M, Somer A, et al. Carbapenem-resistant Klebsiella pneumoniae colonization in pediatric and neonatal intensive care units: risk factors for progression to infection. Braz J Infect Dis 2016;20:134-140.

10. US Department of Health and Human Services/Centers for Disease Control and Prevention. New Delhi metallo- $\beta$-lactamaseproducing carbapenem-resistant Enterobacteriaceae identified in patients without known health care risk factors-Colorado, 2014-2016. MMWR 2016;65:1414-1415.

\section{Hypervirulent Clostridium difficile Strain Has Arrived in Brazil}

To the Editor-Clostridium difficile is an important cause of diarrhea in hospitals all over the world. It is among the most common organisms related to healthcare-associated infections in the United States and represents a serious public health concern. ${ }^{1}$ Clinical manifestations of $C$. difficile infection (CDI) range from asymptomatic carriage, mild or moderate diarrhea, to fulminant colitis. ${ }^{2}$

In the last 20 years, hypervirulent isolates of $C$. difficile have been increasingly reported, mostly in North America and Europe. ${ }^{1}$ The main hypervirulent strain was named ribotype 027 (North American BI, NAP1/027). This epidemic strain was also reported in Asia, providing evidence of worldwide spread. ${ }^{3}$ Patients infected with the 027 strain are 3 times more likely to have severe disease than those infected with non-027 strains. ${ }^{4,5}$ This finding was linked to increased production of $\mathrm{A}, \mathrm{B}$, and binary toxins, in association with a mutation in the gene regulating of the expression of these toxins, leading to overproduction. However, few studies have investigated the presence of hypervirulent strains of $C$. difficile in developing countries, particularly in Latin America. ${ }^{6,7}$ Here, we make the first report of the detection of the hypervirulent $C$. difficile strain in Brazil.

\section{METHODS}

\section{Setting and Patients}

The hypervirulent $C$. difficile strain was noticed during the conduction of a point-prevalence multicenter study in Brazil. Fecal samples were tested with a commercial real-time PCR kit (Xpert C. difficile test; Cepheid, Sunnyvale, CA) in accordance with the manufacturer's instructions. In addition, samples were also submitted to $C$. difficile culture, using absolute alcohol at room temperature, subcultured in CM0601 C. difficile agar (Oxoid), enriched with 7\% blood horse, D-cycloserine, and cefoxitin for 48 hours under an anaerobic atmosphere. Species identification of suspected colonies was confirmed by matrix-assisted laser desorption/ionization (Brucker Daltonics, Bremen, Germany).

Presumptive diagnosis of infection with the 027 strain was conducted with GeneXpert and confirmed with in-house polymerase chain reaction (PCR), designed to amplify the $t c d A$ and $t c d B$ toxin genes and $t c d C$ negative regulator toxin gene. ${ }^{10}$ The PCR products were purified using the enzyme Exo-SAPIT (Thermo Fischer, Waltham, MA) and sequenced using the BigDye Terminator version 3.1 Cycle Sequencing Kit (Life Technology, Carlsbad, CA). Clostridium difficile ATCC 9689 was used as a control. Sequence type was determined by multilocus sequencing typing, ${ }^{10}$ and an online database was used to assign sequence typing (http://pubmlst.org/cdifficile).

\section{RESULTS}

The index case with a GeneXpert 027 positive result was recovered from a 68-year-old man who had a 2-month history of diarrhea and was heavily exposed to antimicrobial drugs (ie, vancomycin, metronidazole, amikacin and meropenem). He was admitted to a university hospital in Porto Alegre, Southern Brazil. At the time of sample collection, the patient had a C-reactive protein level of $109.7 \mathrm{mg} / \mathrm{L}$ and a leukocyte count of 8,000 cells $/ \mathrm{mL}$. The isolate harbored $t c d A$ and $t c d B$ toxin genes and the $t c d C$ deletion (frameshift deletion, 18 nucleotides) that indicates toxin overproduction (Figure 1). The isolate was classified as ST67, belonging to clade 2. This clade contains a high diversity of sequence typing that also includes ST1 (NAP1/027).

In another hospital located in the same city 1 month later, we identified another case of ribotype 027 using GeneXpert. Unfortunately, this case occurred in an outpatient, and we were unable to recover any sample for DNA sequencing. The patient had been using the following antibiotics $>1$ month for a difficult-to-treat otitis: amoxicillin, amoxicillin/clavulanate, and cefuroxime. She arrived in the emergency room with watery diarrhea lasting for 7 days. In the 3 days before admission, she reported abdominal pain, nausea, and malaise. Blood tests revealed 15,580 leucocytes/mL (81.4\% neutrophils) and 349,000 platelets/mL. Abdominal ultrasound showed signs of colitis. The patient was discharged with metronidazole and was lost to follow-up. 


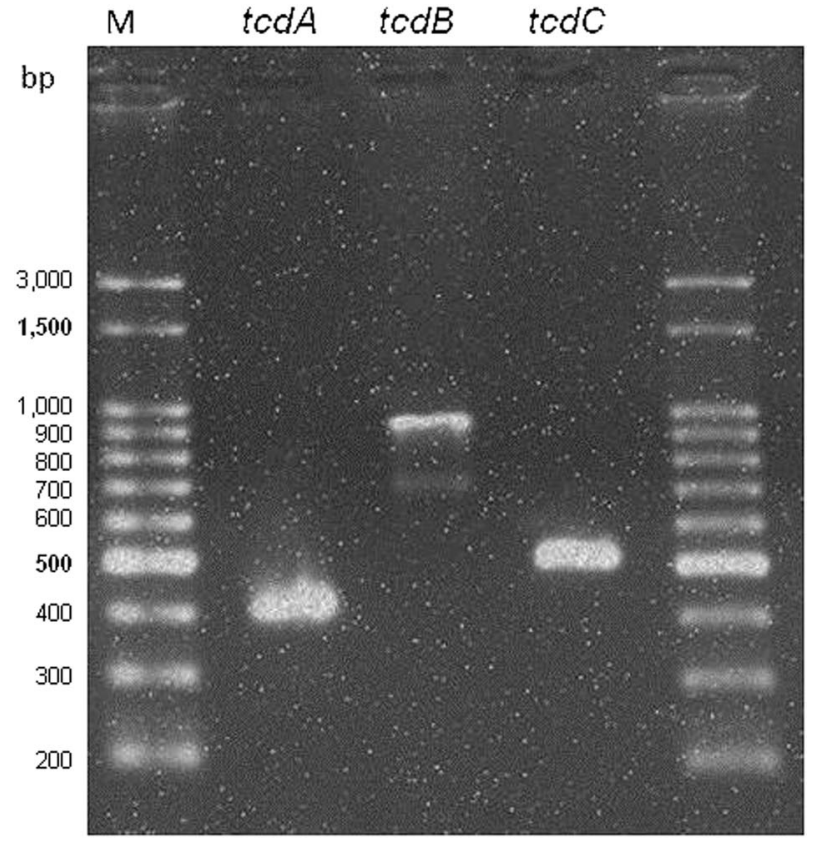

FIGURE 1. In-house polymerase chain reaction (PCR) test results for toxin genes $t c d \mathrm{~A}, t c d \mathrm{~B}$, and the negative promoter toxin gene $t c d$ C. Legend: M, 100-bp size DNA ladder.

\section{I S C USS I O N}

A diagnosis of CDI is based on a combination of clinical history and laboratory detection of $C$. difficile toxins in the feces or cultured isolates. Nucleic acid amplification-based tests offer higher sensitivity and specificity. Although widely used for CDI diagnosis in developed countries, widespread implementation of such technologies in developing countries is still hampered by cost-related issues, in addition to the need of qualified infrastructure and staff. ${ }^{7}$ In Brazil, immune enzymatic detection of $C$. difficile toxins remains the most widely used technique for CDI diagnosis because it is relatively inexpensive and easy to perform.

Diagnosis by GeneXpert offers a number of advantages, including agility, accuracy and presumptive detection of the hypervirulent strains with $\sim 90 \%$ specificity. ${ }^{8}$ On the other hand, a study reported unusual Xpert amplification curves from stool samples presumptively identified as 027/NAP1/BI but not confirmed by PCR ribotyping and $t c d C$ gene sequencing. ${ }^{9}$ Also, in places with a low prevalence of hypervirulent strains, the positive predictive value of the test may be further reduced, reinforcing the importance of performing confirmatory tests. However, molecular typing of $C$. difficile isolates may not be available in all laboratories, and multiple strains of $C$. difficile may coexist in fecal samples.

The confirmed occurrence of hypervirulent $C$. difficile strain in Brazil emphasizes the need to improve surveillance strategies to potentially avoid outbreaks. Recently, Costa Rica, Chile, and Panama also reported isolation of the hypervirulent strain. ${ }^{3}$ Ultimately, cost-effectiveness studies evaluating the use of real-time
PCR to rapidly screen $C$. difficile infections are needed in Latin America. A recent study $\left(69^{\text {th }}\right.$ ACC Annual Scientific Meeting, 2017, Abstract B-098) cited 10 cases of circulating 027 strains in the state of São Paulo, Brazil, but their finding was not confirmed by DNA sequencing and no epidemiological data were provided. Our data highlight the importance of testing for hypervirulence strains of $C$. difficile in patients diagnosed with CDI.

\section{ACKNOWLEDGMENTS}

Financial support: No financial support was provided relevant to this article.

Potential conflicts of interest: All authors report no conflicts of interest relevant to this article.

\author{
Renata N. Pires, RN; ${ }^{1,2}$ \\ Alexandre A. Monteiro, BSc; ${ }^{1}$ \\ Gabriele Z. Saldanha, BSc; ${ }^{3}$ \\ Diego R. Falci, MD; ${ }^{1,4,5}$ \\ Cassia Ferreira B. Caurio, BSc; ${ }^{1}$ \\ Teresa Cristina T. Sukiennik, MD; ${ }^{2}$ \\ Franciele C. Adam, BSc; ${ }^{3}$ \\ Alessandro C. Pasqualotto, MD; ${ }^{1,2}$ \\ Andreza F. Martins, PharmD ${ }^{3}$
}

Affiliations: 1. Universidade Federal de Ciências da Saúde De Porto Alegre, Porto Alegre, Brazil; 2. Santa Casa de Misericórdia de Porto Alegre, Porto Alegre, Brazil; 3. Universidade Federal do Rio Grande do Sul, Porto Alegre, Brazil; 4. Hospital de Clínicas de Porto Alegre, Porto Alegre, Brazil; 5. Universidade La Salle, Canoas, Brazil.

Address correspondence to Alessandro C. Pasqualotto, MD, PhD, Molecular Biology Laboratory, Av Independencia 155, Hospital Dom Vicente Scherer Heliponto, Porto Alegre 90150035 Brazil (pasqualotto@santacasa.tche.br). Infect Control Hosp Epidemiol 2018;39:371-373

(C) 2018 by The Society for Healthcare Epidemiology of America. All rights reserved. 0899-823X/2018/3903-0026. DOI: 10.1017/ice.2017.280

\section{REFERENCES}

1. Di Bella S, Paglia MG, Johnson E, Petrosillo N. Clostridium difficile 027 infection in Central Italy. BMC Infect Dis 2012; 12:370.

2. Peng Z, Liu S, Meng X, et al. Genome characterization of a novel binary toxin-positive strain of Clostridium difficile and comparison with the epidemic 027 and 078 strains. Gut Pathog 2017;9:42.

3. Aguayo C, Flores R, Lévesque S, et al. Rapid spread of Clostridium difficile NAP1/027/ST1 in Chile confirms the emergence of the epidemic strain in Latin America. Epidemiol Infect 2015;143: 3069-3073.

4. Leffler DA, Lamont JT. Clostridium difficile infection. N Engl J Med. 2015;372:1539-1548.

5. Miller M, Gravel D, Mulvey M, et al. Health care-associated Clostridium difficile infection in Canada: patient age and infecting strain type are highly predictive of severe outcome and mortality. Clin Infect Dis 2010;50:194-201.

6. Pires RN, Monteiro AA, Carneiro LC, et al. Clostridium difficile infection in Brazil: A neglected problem? Am J Infect Control 2014;42:459-460. 
7. Monteiro AA, Pires RN, Persson S, Rodrigues Filho EM, Pasqualotto AC. A search for Clostridium difficile ribotypes 027 and 078 in Brazil. Braz J Infect Dis 2014;18:672-674.

8. Chiang D, Ng S, La MV, Jureen R, Lin RTP, Teo JWP. Performance assessment of the BD MAX Cdiff assay in comparison to Xpert $C$. difficile assay in a setting with very low prevalence of toxigenic Clostridium difficile PCR ribotype 027. Anaerobe 2014; 30:156-158.

9. Kok J, Wang Q, Thomas LC, Gilbert GL. Presumptive identification of Clostridium difficile strain 027/NAP1/BI on Cepheid Xpert: interpret with caution. J Clin Microbiol 2011; 49:3719-3721.

10. Griffiths D, Fawley W, Kachrimanidou M, et al. Multilocus sequence typing of Clostridium difficile. J Clin Microbiol 2010;48: 770-778.

\section{Potential Impact of Antibiotic Stewardship Programs on Overall Antibiotic Use in Adult Acute-Care Hospitals in the United States}

To the Editor-We sought to characterize the expected decline in US acute-care antibiotic prescribing resulting from new accreditation standards requiring antibiotic stewardship programs. ${ }^{1}$ We conducted a narrative review of published literature assessing the impact of antibiotic stewardship program implementation on total antibiotic prescribing in acute-care hospitals in the United States.

A PubMed search was performed using the following search strategy: antimicrobial OR antibiotic AND stewardship from January 1996 to December 2016. Finally, 12 articles and 1 abstract that reported the effect of antimicrobial stewardship programs on total antibiotic use in adult US acute-care hospitals were included. The median and interquartile range (IQR) of decline in antibiotic use observed with implementation of antibiotic stewardship programs were calculated (Table 1). If no significant decline in antibiotic use was noted, percentage decline was considered to be zero. To quantify the expected national decline in antibiotic use following the implementation of antibiotic stewardship programs, the calculated median and IQR were applied to the 2012 national estimate of adult antibiotic use in acute care hospitals obtained from the Truven Health MarketScan Hospital Drug Database (HDD). ${ }^{2}$

Most studies reported the implementation of "audit and feedback," with or without antibiotic restriction; 2 studies evaluated the effect of computerized decision support; and 1 study implemented an educational intervention pertaining to the electronic health record. The median decline in antibiotic use was $15.8 \%$ (IQR, $0-27.3 \%$ ). The national estimate of total adult antibiotic use in 2012 in US acute-care hospitals was
103 million days of therapy (DOT) or 817.8 DOT per 1,000 patient days (PD) as reported by the HDD. Widespread implementation of antibiotic stewardship programs would therefore be expected to lead to an estimated median decline of 16 million DOT (IQR, 0-28 million) in total systemic antibiotic use, or a median national target of 688.6 DOT per 1,000 PD in US adult acute-care hospitals.

None of the studies reviewed showed worsening outcomes, such as increased mortality or readmissions, consistent with a recent Cochrane review. ${ }^{3}$ Reporting clinical outcomes provides an opportunity to assess the safety and potential benefits of reducing unnecessary antibiotic use. A few studies showed no change or a decrease in Clostridium difficile infection and antibiotic-resistant pathogens, which are important metrics for assessing the patient-level impact of antibiotic stewardship. ${ }^{4}$

A publication bias toward positive results may lead to the overestimation of the effect of antibiotic stewardship programs. Hospital characteristics and types of interventions varied, as did the approaches to measurement and to reported outcomes. However, with the implementation of antibiotic stewardship programs in adult US acute-care hospitals, an estimated 15.8\% ( 16 million DOT) of total antibiotic prescribing might be avoided. This number does not include other important improvements that could be made, such as narrowing the spectrum of therapy and shortening postdischarge courses. Hospital stewardship programs should be supported in their efforts to protect patients from preventable harms caused by unnecessary antibiotic exposure.

\section{ACKNOWLEDGMENTS}

Financial support: This work was supported by the Centers for Disease Control and Prevention. The findings and conclusions in this report are those of the authors and do not necessarily represent the official position of the Centers for Disease Control and Prevention.

Potential conflicts of interest: All authors report no conflicts of interest relevant to this article.

\section{Sarah Kabbani, MD, MSc; James Baggs, PhD; Lauri A. Hicks, DO; Arjun Srinivasan, MD}

Affiliations: Division of Healthcare Quality Promotion, Centers for Disease Control and Prevention, Atlanta, Georgia.

Address correspondence to Sarah Kabbani, MD, MSc, Medical officer, Office of Antibiotic Stewardship, Division of Healthcare Quality Promotion, Centers for Disease Control and Prevention, 1600 Clifton Road MS A-31, Atlanta, GA 30329 (nfq8@cdc.gov) or to Lauri Hicks, DO, CAPT, US Public Health Service, Director, Office of Antibiotic Stewardship, Division of Healthcare Quality Promotion, Centers for Disease Control and Prevention, 1600 Clifton Road MS A-31, Atlanta, GA 30329 (auq3@cdc.gov).

Infect Control Hosp Epidemiol 2018;39:373-376

(C) 2018 by The Society for Healthcare Epidemiology of America. All rights reserved. 0899-823X/2018/3903-0027. DOI: 10.1017/ice.2017.273 